\title{
Visão de Orientadores e Orientandos sobre o Software Online de Supervisão da Investigação - IARS ${ }^{\circledR}$
}

\author{
Dayse Neri de Souza*, Francislê Neri de Souza*, Isabel Alarcão*, António Moreira* \\ dayneri@ua.pt,fns@ua.pt, ialarcao@ua.pt, moreira@ua.pt \\ * Centro de Investigação Didática e Tecnologia na Formação de Formadores, Universidade de Aveiro, \\ 3810 193, Aveiro, Portugal \\ Departamento de Educação, Universidade de Aveiro, 3810 193, Aveiro, Portugal
}

DOI: 10.17013/risti.e4.66-78

Resumo: No âmbito da pós-graduação, são vários os dilemas e problemas enfrentados na investigação. A qualidade dos projetos e a relação entre orientador e orientando, têm-se revelado como alguns dos grandes desafios. São muitos os estudos que apontam o uso das TIC como um meio de ajuda no processo de investigação e na produção dos resultados com qualidade. Apresentamos neste artigo a percepção de orientadores e orientados que utilizaram o software online IARS $^{\circledR}$ como uma ferramenta de apoio na elaboração e gestão do projeto de investigação. Este estudo é de cariz exploratório de análise descritiva com apoio do SPSS. Os resultados atestam uma percepção positiva e rica acerca da utilização do software recentemente divulgado. Assim, consideraram o IARS $^{\circledR}$ de fácil utilização, agradável, flexível, estimulador, claro e de fácil compreensão em temos organizacionais. Apontam também para as dificuldades nas etapas de construção do projeto e comunicação entre os investigadores, e reconhecem como o sistema pode ser útil neste âmbito.

Palavras-chave: Processo de investigação; TIC; IARS ${ }^{\circledR}$; comunicação; relação orientador-orientando

Views of Supervisors and Supervisees about the Online Software for Research Management - IARS ${ }^{\circledR}$

Abstract: At graduate level, there are several dilemmas and problems faced in research. The quality of the projects and the relationship between supervisor and research student have been shown to be major challenges. There are many studies that show the use of ICT as a means to help in the research process and the production of quality results. We present here the perception of supervisors and research students who used the online software IARS ${ }^{\circledR}$ as a support tool in the development and management of the research project. This study is of exploratory nature and of descriptive analysis with the support of SPSS. The results show a positive and rich perception on using this recently released software. Thus they considered IARS $($ user-friendly, nice, flexible, stimulating, clear and easy to understand in organizational terms. They also point to the difficulties in the construction stages of 
the project and communication between researchers, and recognize how the system can be useful in this context.

Keywords: Research process; ICT; IARS ${ }^{\circledR}$; communication; supervisor-supervisee relationship.

\section{Introdução}

O estudo realizado por Baptista (2011) revela que tem havido profundo interesse em pesquisas relacionadas com o impacto da investigação ao nível da pós-graduação. Este interesse justifica-se pelo aumento do número de estudantes que ingressam na pósgraduação, seja mestrado e/ou doutorado.

Os estudiosos alertam para os enormes desafios que se colocam à definição de critérios e padrões de qualidade na formação em investigação. Em seu estudo, Baptista (2011) destaca o Comunicado de Bergen de 2005, ao afirmar que a investigação no contexto do ensino superior promove o desenvolvimento cultural e económico e também a coesão social no sentido de melhorar a qualidade do processo de ensino e aprendizagem e aumentar a competitividade e atratividade europeia. Seguimos na mesma direção de Baptista (2011) quando a autora se questiona: Quais são as competências que são exigidas aos alunos e supervisores de doutoramento, bem como às equipes de supervisão, para que eles possam conhecer e atingir níveis elevados de qualidade no processo e no produto do doutoramento?

Concordamos com a opinião de alguns autores (Lessing \& Schulze, 2002; Thompson, Kirkman Watson \& Stewart, 2005; Calma, 2007; Agu \& Odimegwu, 2014) quando salientam que o sucesso no processo e produto final da investigação está associado à relação que se estabelece entre o orientando e o orientador. Segundo Lessing e Schulze (2002), o orientador tem um importante papel a exercer no processo de investigação e na relação com o orientando. O orientador deve orientar, aconselhar, dar apoio emocional e garantir a qualidade científica. Nesta direção, Thompson, Kirkman, Watson e Stewart (2005) salientam que os orientadores devem partilhar a sua competência intelectual, de maneira a aumentar a autoconfiança e autoestima dos orientandos. Numa linha concordante, acreditamos que o supervisor deve assumir-se como um facilitador, crítico intelectual, conselheiro, atencioso, disponível, amigo, solidário e entusiasta. Esses atributos podem proporcionar uma relação de confiança, respeito e tolerância.

Sidhu, Kaur, Fook e Yunus (2014) defendem também que a atitude dos orientadores em relação aos seus orientandos é crucial durante a orientação. Ou seja, quando os orientadores criam sentimentos negativos, atitudes negativas junto dos seus orientandos, poderão impedi-los de produzir. No mesmo ponto de vista dos autores, julgamos que, quando os supervisores são eficazes no processo de orientação, ajudam os orientandos a obter sucesso. O processo de investigação é um desafio para todos os cientistas, mas especialmente para os jovens investigadores. Estes jovens têm incertezas, dilemas e problemas, mas segundo Alarcão (2014), os dilemas dos jovens investigadores podem ser transformados em problemas epistémicos com solução viável.

Face às preocupações sobre o nível de qualidade da orientação na pós-graduação, têm emergido estudos sobre a necessidade de definir meios ou modelos que facilitem o processo de orientação/supervisão e interação entre os orientadores e seus orientandos 
(Agu \& Odimegwu, 2014; Mainhard, Van der Rijst \& Wubbels, 2009; Neri de Souza, Neri de Souza \& Costa, 2014).

Vários são os Modelos já estudados e analisados pelos especialistas (Alarcão, 2014; Gurr, 2001; Dysthe, 2002). No entanto, o mais conhecido e tradicionalmente adotado face-to-face - pode ser complementado com o uso das TIC. O modelo conhecido como misto - blended - tem sido adotado por muitos orientadores, tendo em conta o número elevado de atividades que os orientadores realizam. Beer \& Mason (2009) apontam para o uso do modelo Blended Learning. Acredita-se que este modelo tem contribuído para melhorar o processo de orientação, de forma a diminuir a carga de trabalho e cooperar na criação de um registo dinâmico no processo de orientação. Ou seja, há a possibilidade de uma combinação entre o presencial e o interativo com o uso das TIC (Escudeiro \& Bidarra, 2008; Agu \& Odimegwu, 2014).

O modelo b-learning, sugerido pelos autores, indica o papel do supervisor como principal fonte de informação num momento presencial, e as consultas à internet e às bibliotecas como uma etapa complementar. Este modelo foi considerado pertinente pelo facto de poder aliviar a sobrecarga dos orientadores, pelo que estamos em sintonia com estes e outros autores ao propor um modelo que está na base do software IARS $₫$ que facilita o apoio no planeamento e execução do percurso investigativo.

\subsection{O Isabel Alarcão Research Software - IARS $®$}

O IARS ${ }^{\circledR}$ foi desenvolvido a partir do guião orientador da construção do plano de investigação, elaborado por Isabel Alarcão na década de 90, enquanto docente de Seminários de Investigação, no âmbito de Mestrados no antigo Departamento de Didática e Tecnologia Educativa da Universidade de Aveiro.

O software é uma aplicação informática de apoio ao processo de orientação de trabalhos de pesquisa académica disponível num ambiente imersivo, distribuído e seguro (Cloud Computing), acedido pela internet. Com esta aplicação Web (www.iars.com), o orientador pode fomentar a interação num ambiente de trabalho tutorial individualizado e eticamente protegido. Permite também trabalhar com grupos e, deste modo, rentabilizar interações menos individualizadas.

Neste âmbito, consideramos o processo de investigação como: i) multifacetado, ii) iterativo e iii) interativo, sendo que o "processo" pressupõe que seja realizado no tempo, por fases multirelacionadas. O ser "multifacetado" deve levar em consideração as diversas abordagens, atores, variáveis e condicionantes do fazer ciência. A dimensão "iterativo" considera que o processo é dinâmico e cíclico nas várias fases da investigação. Para além disso, todo este processo ocorre na "interação" humana entre orientador e orientandos e diversos sujeitos colaboradores no fazer ciência (ver Fig.1).

O IARS ${ }^{\circledR}$ assenta nos seguintes pressupostos:

- $\quad$ organizador conceptual do projeto nas suas fases;

- matriz aberta suscetível de ser utilizada com diferentes abordagens metodológicas;

- $\quad$ estrutura baseada em questões organizadoras e estimuladoras do pensamento do investigador; 


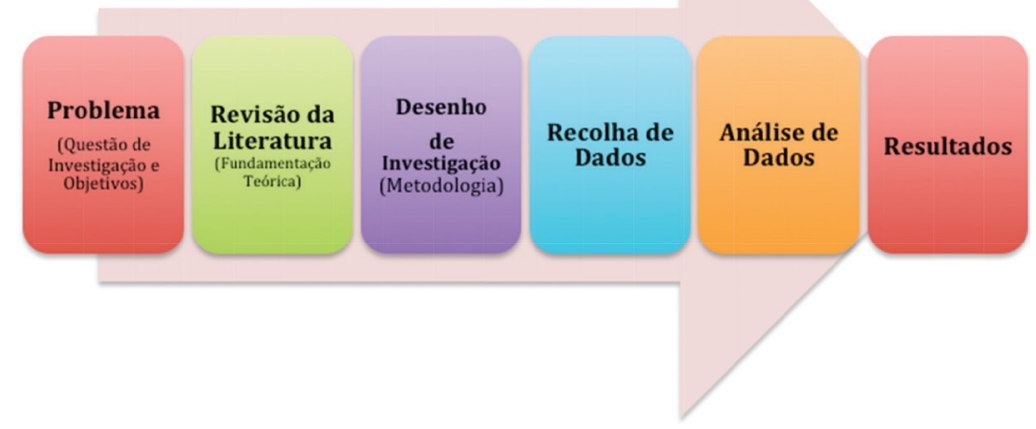

Figura 1 - Processo Genérico de Investigação

- $\quad$ simplicidade de utilização;

- interação orientando/orientador;

- registo da evolução dos orientandos em jeito de "portfólio de investigação";

- organizador sistemático do projeto e dos resultados da investigação.

A aplicação é composta por uma área "Saber Mais", que fornece ao pesquisador ajuda na elaboração dos projetos. Esta área é, porém, muito discreta, pois não pretende nem deve substituir-se ao orientador real. É também generalista uma vez que o presente software pode ser utilizado com diferentes paradigmas metodológicos.

\section{Metodologia}

Este artigo se emoldura numa metodologia de investigação e desenvolvimento de uma plataforma online para a gestão colaborativa de projetos de investigação, principalmente para as ciências humanas e sociais, educação e diversas outras áreas: o IARS.

Com o propósito de avaliarmos a percepção de orientadores e orientandos que participaram de vários workshops sobre o software IARS®, foi elaborado um questionário de auto resposta com questões abertas e fechadas. Esteve disponível em formato online para que os participantes pudessem responder após o encerramento dos Workshops. O questionário é composto por quatro partes. Na primeira são solicitados os dados pessoais dos participantes. Na segunda, questões acerca: i) da opinião dos participantes sobre o ato de investigar, e ii) da relação interpessoal e da gestão no desenvolvimento do projeto de investigação. A terceira parte é sobre o software (interface, navegação, dificuldades, aspetos positivos e negativos). A quarta e última parte consiste em espaços em que os participantes podem deixar comentários, referir-se às características do IARS ${ }^{\circledR}$ e à sua relação com a investigação individual e apresentar sugestões de melhorias para o software.

A duração da formação tinha aproximadamente duas horas e meia, a contar com momentos de esclarecimentos e dúvidas pontuais acerca da utilização do IARS ${ }^{\circledR}$. Os objetivos principais do Workshop foram: 
a) Conhecer a organização conceptual de um projeto de investigação; b) identificar as questões conceptuais, organizadoras e estimuladoras para a elaboração de um projeto de investigação; c) consciencializar para uma maior e melhor interação entre orientando e orientador no uso do IARS ${ }^{\circledR} ;$ d) utilizar o IARS ${ }^{\circledR}$ como um organizador sistemático do projeto e dos resultados da investigação; e) desenvolver, com o uso do IARS ${ }^{\circledR}$, capacidades e atitudes de investigação que facilitem a elaboração de trabalhos científicos; f) aferir, com o uso do $\mathrm{IARS}^{\circledR}$, uma maior coerência interna nas etapas de elaboração do projeto de investigação; g) conhecer a estrutura funcional do IARS ${ }^{\circledR}$ nas suas diferentes abordagens metodológicas e aplicar as suas potencialidades na elaboração das etapas de um projeto de investigação.

Neste estudo que agora apresentamos, de caráter exploratório, primamos por uma análise descritiva acerca das perceções dos 87 sujeitos realizada com o apoio do SPSS 21.o. Apresentamos os resultados obtidos a partir da análise geral das questões fechadas através de frequências e percentagens.

\section{Resultados}

Os sujeitos dos Workshops foram 20.5\% $(\mathrm{N}=18)$ do género masculino e $78.4 \%(\mathrm{~N}=69)$ feminino. Estavam fazendo licenciatura (43.2\%), mestrado (28.4\%) ou doutoramento (22.7\%) e pós-doutoramento (4.5\%). No gráfico 1 comparamos o número de participantes de acordo com as funções de investigação que exerciam na altura em que responderam ao questionário no final dos Workshops. Como se pode verificar a maior parte eram orientandos (65.5\%), 22,7\% se identificaram somente como orientadores, e 10,3\% como orientadores fazendo mestrado, doutoramento ou pós-douramento.

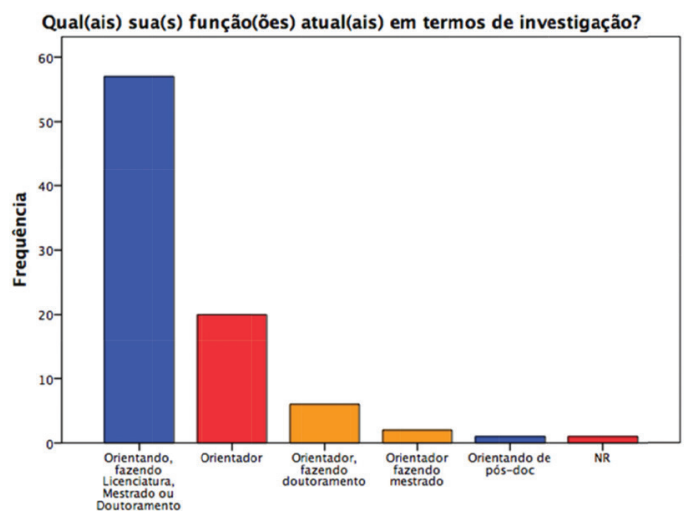

Gráfico 1 - Distribuição dos participantes dos Workshops IARS ${ }^{\circledR}$ por função na investigação

Como o $\operatorname{IARS}^{\circledR}$ é uma plataforma de gestão do processo de desenvolvimento do projeto de investigação, é importante conhecer a visão dos utilizadores (orientadores e orientandos) sobre diversas dimensões, como: i) o ato de investigar, ii) elaboração e execução do projeto, iii) processo de comunicação na orientação e iv) gestão das tarefas e calendário de desenvolvimento do projeto. A seguir iremos apresentar cada uma destas dimensões, expressas pelos participantes numa escala de concordância. 
Na Tabela 1 apresentamos as respostas dos sujeitos em relação a várias dimensões sobre o ato de investigar: i) comunicação, ii) negociação, iii) resiliência, iv) autonomia, v) rigor e vi) trabalho em grupo. Houve concordância no padrão de respostas, tanto dos orientadores como dos orientandos.

O maior nível de discordância está no primeiro item da Tabela 1, sobre o processo de comunicação (28,7\%), sendo que neste item $17,2 \%$ não têm opinião formada sobre o assunto. Mesmo assim, os participantes concordam (54\%) que, se o processo de comunicação falhar, colocará em causa a conclusão da investigação. No entanto, consideram também a importância do processo de negociação na investigação (86.2\%), que é um aspeto fundamental relacionado à comunicação.

\begin{tabular}{|c|c|c|c|c|c|}
\hline & $\begin{array}{l}\text { Discordo } \\
\text { totalmente }\end{array}$ & Discordo & $\begin{array}{l}\text { Sem } \\
\text { opinião }\end{array}$ & Concordo & $\begin{array}{l}\text { Concordo } \\
\text { totalmente }\end{array}$ \\
\hline $\begin{array}{l}\text { 1. Se o processo de comunicação } \\
\text { entre orientador(es) e } \\
\text { orientado(s) falhar não é possível } \\
\text { concluir a investigação. }\end{array}$ & $\begin{array}{l}5 \\
5,7 \%\end{array}$ & $\begin{array}{l}20 \\
23,0 \%\end{array}$ & $\begin{array}{l}15 \\
17,2 \%\end{array}$ & $\begin{array}{l}28 \\
32,2 \%\end{array}$ & $\begin{array}{l}19 \\
21,8 \%\end{array}$ \\
\hline $\begin{array}{l}\text { 2. Os orientandos deveriam negociar } \\
\text { com o(s) orientador(es) as opções } \\
\text { em termos da investigação. }\end{array}$ & $\begin{array}{l}1 \\
1,1 \%\end{array}$ & $\begin{array}{l}3 \\
3,4 \%\end{array}$ & $\begin{array}{l}8 \\
9,2 \%\end{array}$ & $\begin{array}{l}41 \\
47,1 \%\end{array}$ & $\begin{array}{l}34 \\
39,1 \%\end{array}$ \\
\hline $\begin{array}{l}\text { 3. Os estudantes deveriam } \\
\text { ter resiliência emocional } \\
\text { para realizar a investigação. }\end{array}$ & $\begin{array}{l}\mathrm{o} \\
\mathrm{o}, 0 \%\end{array}$ & $\begin{array}{l}1 \\
1,1 \%\end{array}$ & $\begin{array}{l}15 \\
17,2 \%\end{array}$ & $\begin{array}{l}46 \\
52,9 \%\end{array}$ & $\begin{array}{l}25 \\
28,7 \%\end{array}$ \\
\hline $\begin{array}{l}\text { 4. Os estudantes deveriam } \\
\text { ser autónomos nas suas } \\
\text { investigações. }\end{array}$ & $\begin{array}{l}\mathrm{o} \\
\mathrm{o}, 0 \%\end{array}$ & $\begin{array}{l}11 \\
12,6 \%\end{array}$ & $\begin{array}{l}12 \\
13,8 \%\end{array}$ & $\begin{array}{l}42 \\
48,3 \%\end{array}$ & $\begin{array}{l}22 \\
25,3 \%\end{array}$ \\
\hline $\begin{array}{l}\text { 5. O(s) orientador }(\mathrm{es}) \text { deveria }(\mathrm{m}) \\
\text { ser rigoroso(s) e exigente(s) } \\
\text { para com a investigação dos seus } \\
\text { estudantes. }\end{array}$ & $\begin{array}{l}\mathrm{o} \\
\mathrm{o}, \mathrm{O} \%\end{array}$ & $\begin{array}{l}1 \\
1,1 \%\end{array}$ & $\begin{array}{l}8 \\
9,2 \%\end{array}$ & $\begin{array}{l}37 \\
42,5 \%\end{array}$ & $\begin{array}{l}41 \\
47,1 \%\end{array}$ \\
\hline $\begin{array}{l}\text { 6. } \mathrm{O}(\mathrm{s}) \text { orientador(es) deveriam } \\
\text { promover a autonomia do } \\
\text { estudante. }\end{array}$ & $\begin{array}{l}\mathrm{o} \\
\mathrm{o}, 0 \%\end{array}$ & $\begin{array}{l}2 \\
2,3 \%\end{array}$ & $\begin{array}{l}6 \\
6,9 \%\end{array}$ & $\begin{array}{l}39 \\
44,8 \%\end{array}$ & $\begin{array}{l}40 \\
46,0 \%\end{array}$ \\
\hline $\begin{array}{l}\text { 7. } \mathrm{O}(\mathrm{s}) \text { orientador(es) deveriam } \\
\text { inserir o estudante num } \\
\text { grupo de investigação. }\end{array}$ & $\begin{array}{l}2 \\
2,3 \%\end{array}$ & $\begin{array}{l}3 \\
3,4 \%\end{array}$ & $\begin{array}{l}21 \\
24,1 \%\end{array}$ & $\begin{array}{l}35 \\
40,2 \%\end{array}$ & $\begin{array}{l}26 \\
29,9 \%\end{array}$ \\
\hline
\end{tabular}

Tabela 1 - Visão Sobre o Ato de Investigar

Os sujeitos foram também questionados sobre as dificuldades encontradas nas fases e tarefas de investigação (Ver Tabela 2), tendo em conta este ser um dos aspectos em que a utilização do IARS ${ }^{\circledR}$ pode contribuir. 


\begin{tabular}{|c|c|c|c|c|c|}
\hline & $\mathbf{1}^{*}$ & 2 & 3 & 4 & 5 \\
\hline $\begin{array}{l}\text { 1. O capítulo das conclusões é a parte mais } \\
\text { exigente de uma investigação. }\end{array}$ & $\begin{array}{l}2 \\
2,3 \%\end{array}$ & $\begin{array}{l}21 \\
24,1 \%\end{array}$ & $\begin{array}{l}19 \\
21,8 \%\end{array}$ & $\begin{array}{l}33 \\
37,9 \%\end{array}$ & $\begin{array}{l}12 \\
13,8 \%\end{array}$ \\
\hline $\begin{array}{l}\text { 2. A análise de dados é a parte mais } \\
\text { exigente de uma investigação. }\end{array}$ & $\begin{array}{l}0 \\
0,0 \%\end{array}$ & $\begin{array}{l}12 \\
13,8 \%\end{array}$ & $\begin{array}{l}11 \\
12,6 \%\end{array}$ & $\begin{array}{l}45 \\
51,7 \%\end{array}$ & $\begin{array}{l}19 \\
21,8 \%\end{array}$ \\
\hline $\begin{array}{l}\text { 3. A recolha de dados é a parte mais } \\
\text { exigente de uma investigação. }\end{array}$ & $\begin{array}{l}0 \\
0,0 \%\end{array}$ & $\begin{array}{l}25 \\
28,7 \%\end{array}$ & $\begin{array}{l}14 \\
16,1 \%\end{array}$ & $\begin{array}{l}40 \\
46,0 \%\end{array}$ & $\begin{array}{l}8 \\
9,2 \%\end{array}$ \\
\hline $\begin{array}{l}\text { 4. Manter a coerência interna de todos as } \\
\text { partes de um projeto é o mais exigente no } \\
\text { processo investigativo. }\end{array}$ & $\begin{array}{l}1 \\
1,1 \%\end{array}$ & $\begin{array}{l}4 \\
4,6 \%\end{array}$ & $\begin{array}{l}7 \\
8,0 \%\end{array}$ & $\begin{array}{l}40 \\
46,0 \%\end{array}$ & $\begin{array}{l}35 \\
40,2 \%\end{array}$ \\
\hline $\begin{array}{l}\text { 5. A parte mais exigente na elaboração } \\
\text { de um projeto é a definição da } \\
\text { fundamentação teórica. }\end{array}$ & $\begin{array}{l}\mathrm{O} \\
\mathrm{O}, \mathrm{O} \%\end{array}$ & $\begin{array}{l}21 \\
24,1 \%\end{array}$ & $\begin{array}{l}11 \\
12,6 \%\end{array}$ & $\begin{array}{l}47 \\
54,0 \%\end{array}$ & $\begin{array}{l}8 \\
9,2 \%\end{array}$ \\
\hline $\begin{array}{l}\text { 6. A parte mais exigente na elaboração } \\
\text { de um projeto é a definição dos } \\
\text { paradigmas e metodologias. }\end{array}$ & $\begin{array}{l}1 \\
1,1 \%\end{array}$ & $\begin{array}{l}10 \\
11,5 \%\end{array}$ & $\begin{array}{l}13 \\
14,9 \%\end{array}$ & $\begin{array}{l}56 \\
64,4 \%\end{array}$ & $\begin{array}{l}7 \\
8,0 \%\end{array}$ \\
\hline $\begin{array}{l}\text { 7. A parte mais exigente na elaboração de } \\
\text { um projeto é a definição das questões } \\
\text { de investigação. }\end{array}$ & $\begin{array}{l}0 \\
0,0 \%\end{array}$ & $\begin{array}{l}12 \\
13,8 \%\end{array}$ & $\begin{array}{l}4 \\
4,6 \%\end{array}$ & $\begin{array}{l}41 \\
47,1 \%\end{array}$ & $\begin{array}{l}30 \\
34,5 \%\end{array}$ \\
\hline
\end{tabular}

* 1. Discordo totalmente, 2. Discordo, 3. Sem opinião, 4. Concordo, 5. Concordo totalmente

Tabela 2 - Visão sobre as fases e tarefas de investigação

Somando o nível de concordância por cada variável declarada de maior dificuldade ou exigência, de forma decrescente, temos: i) coerência interna (86\%), ii) questões de investigação (81.6\%), iii) análise de dados (73.5\%), iv) paradigmas e metodologias (72.4\%), v) fundamentação teórica (63.2\%), vi) recolha de dados (55.2\%), e vii) conclusões $(51,7 \%)$. É exatamente nos aspetos em que a grande maioria dos sujeitos declararam existir maior dificuldade e/ou exigência que o IARS ${ }^{\circledR}$ pretende ser um apoio mais imediato no desenvolvimento do projeto e execução das tarefas.

\subsection{Gestão e Desenvolvimento do Projeto de Investigação}

Um projeto de investigação é uma atividade de grande complexidade que requer um grupo de trabalho, mesmo que este grupo seja apenas um orientador e seu orientando. Gerir o seu desenvolvimento implica desafios cognitivos e emocionais. Inspirado no questionamento de Phillips e Derek (2005) perguntámos aos sujeitos dos Workshops IARS $^{\circledR}$ sobre o que esperam os orientadores dos seus orientandos e o que esperam os orientandos dos seus orientadores, e também quais os deveres e tarefas de cada um deles. Na Tabela 3, até à frase 10 incide-se sobre a opinião sobre o que os estudantes deveriam fazer, desenvolver e/ou tomar de iniciativa. Da frase 11 em diante deslocalizase o foco para as iniciativas que os orientadores deveriam tomar na investigação. 


\begin{tabular}{|c|c|c|c|c|c|}
\hline & $\mathbf{1}^{*}$ & 2 & 3 & 4 & 5 \\
\hline $\begin{array}{l}\text { 1. Os estudantes deveriam informar sempre o(s) } \\
\text { orientador(es) das suas atividades de investigação. }\end{array}$ & $\begin{array}{l}1 \\
1,1 \%\end{array}$ & $\begin{array}{l}4 \\
4,6 \%\end{array}$ & $\begin{array}{l}4 \\
4,6 \%\end{array}$ & $\begin{array}{l}37 \\
42,5 \%\end{array}$ & $\begin{array}{l}41 \\
47,1 \%\end{array}$ \\
\hline $\begin{array}{l}\text { 2. Os estudantes deveriam ler e partilhar as } \\
\text { referências bibliográficas. }\end{array}$ & $\begin{array}{l}\mathrm{O} \\
\mathrm{O}, \mathrm{O} \%\end{array}$ & $2,3 \%$ & $\begin{array}{l}5 \\
5,7 \%\end{array}$ & $\begin{array}{l}51 \\
58,6 \%\end{array}$ & $\begin{array}{l}29 \\
33,3 \%\end{array}$ \\
\hline $\begin{array}{l}\text { 3. Os estudantes deveriam solicitar sempre } \\
\text { referências bibliográficas ao(s) orientador(es). }\end{array}$ & $\begin{array}{l}\mathrm{O} \\
\mathrm{O}, \mathrm{O} \%\end{array}$ & $\begin{array}{l}10 \\
11,5 \%\end{array}$ & $\begin{array}{l}13 \\
14,9 \%\end{array}$ & $\begin{array}{l}47 \\
54,0 \%\end{array}$ & 17 \\
\hline $\begin{array}{l}\text { 4. Os estudantes deveriam desenvolver } \\
\text { competências e habilidades de investigador } \\
\text { procurando realizar formações. }\end{array}$ & $\begin{array}{l}0 \\
0,0 \%\end{array}$ & $\begin{array}{l}0 \\
0,0 \%\end{array}$ & $\begin{array}{l}8 \\
9,2 \%\end{array}$ & $\begin{array}{l}51 \\
58,6 \%\end{array}$ & $\begin{array}{l}28 \\
32,2 \%\end{array}$ \\
\hline $\begin{array}{l}\text { 5. Os estudantes deveriam desenvolver competências } \\
\text { reflexivas e de questionamento. }\end{array}$ & $\begin{array}{l}0 \\
0,0 \%\end{array}$ & $\begin{array}{l}\mathrm{O} \\
\mathrm{O}, \mathrm{O} \%\end{array}$ & $1,1 \%$ & $\begin{array}{l}32 \\
36,8 \%\end{array}$ & $\begin{array}{l}54 \\
62,1 \%\end{array}$ \\
\hline $\begin{array}{l}\text { 6. Os estudantes deveriam ter disciplina pessoal } \\
\text { para realizar a investigação. }\end{array}$ & $\begin{array}{l}0 \\
0,0 \%\end{array}$ & $\begin{array}{l}0 \\
0,0 \%\end{array}$ & $\begin{array}{l}2 \\
2,3 \%\end{array}$ & $\begin{array}{l}40 \\
46,0 \%\end{array}$ & $\begin{array}{l}45 \\
51,7 \%\end{array}$ \\
\hline $\begin{array}{l}\text { 7. Os estudantes deveriam demonstrar iniciativa } \\
\text { própria na investigação. }\end{array}$ & $\begin{array}{l}0 \\
0,0 \%\end{array}$ & $1,1 \%$ & $\begin{array}{l}3 \\
3,4 \%\end{array}$ & $\begin{array}{l}39 \\
44,8 \%\end{array}$ & $\begin{array}{l}44 \\
50,6 \%\end{array}$ \\
\hline $\begin{array}{l}\text { 8. Os estudantes deveriam seguir os conselhos } \\
\text { do(s) orientador(es). }\end{array}$ & $\begin{array}{l}0 \\
0,0 \%\end{array}$ & $\begin{array}{l}1 \\
1,1 \%\end{array}$ & $7,8,0 \%$ & $\begin{array}{l}48 \\
55,2 \%\end{array}$ & $\begin{array}{l}31 \\
35,6 \%\end{array}$ \\
\hline $\begin{array}{l}\text { 9. Os estudantes deveriam solicitar reuniões } \\
\text { regulares com o(s) orientador(es). }\end{array}$ & $\begin{array}{l}0 \\
0,0 \%\end{array}$ & $\begin{array}{l}3 \\
3,4 \%\end{array}$ & $\begin{array}{l}6 \\
6,9 \%\end{array}$ & $\begin{array}{l}55 \\
63,2 \%\end{array}$ & $\begin{array}{l}23 \\
26,4 \%\end{array}$ \\
\hline $\begin{array}{l}\text { 10. Os estudantes deveriam fazer relatórios } \\
\text { regulares ao seu(s) orientador(es) sobre o } \\
\text { desenvolvimento da investigação. }\end{array}$ & $\begin{array}{l}0 \\
0,0 \%\end{array}$ & 13 & $\begin{array}{l}9 \\
10,3 \%\end{array}$ & $\begin{array}{l}45 \\
51,7 \%\end{array}$ & $\begin{array}{l}20 \\
23,0 \%\end{array}$ \\
\hline $\begin{array}{l}\text { 11. } \mathrm{O}(\mathrm{s}) \text { orientador(es) deveria }(\mathrm{m}) \text { estimular seus } \\
\text { estudantes a publicarem suas investigações. }\end{array}$ & $\begin{array}{l}0 \\
0,0 \%\end{array}$ & $\begin{array}{l}\mathrm{O} \\
\mathrm{O}, \mathrm{O} \%\end{array}$ & $4,6 \%$ & $\begin{array}{l}41 \\
47,1 \%\end{array}$ & $\begin{array}{l}42 \\
48,3 \%\end{array}$ \\
\hline $\begin{array}{l}\text { 12. } \mathrm{O}(\mathrm{s}) \text { orientador(es) deveria }(\mathrm{m}) \text { promover uma } \\
\text { articulação entre os trabalhos de outros estudantes. }\end{array}$ & $1,1 \%$ & $\begin{array}{l}4 \\
4,6 \% \\
\end{array}$ & $\begin{array}{l}18 \\
20,7 \%\end{array}$ & $\begin{array}{l}40 \\
46,0 \%\end{array}$ & $\begin{array}{l}24 \\
27,6 \%\end{array}$ \\
\hline $\begin{array}{l}\text { 13. } \mathrm{O}(\mathrm{s}) \text { orientador(es) deveria }(\mathrm{m}) \text { ler } \\
\text { previamente os trabalhos dos estudantes. }\end{array}$ & $\begin{array}{l}0 \\
0,0 \%\end{array}$ & $1,1 \%$ & $\begin{array}{l}2 \\
2,3 \%\end{array}$ & $\begin{array}{l}39 \\
44,8 \%\end{array}$ & $\begin{array}{l}45 \\
51,7 \%\end{array}$ \\
\hline $\begin{array}{l}\text { 14. } \mathrm{O}(\mathrm{s}) \text { orientador(es) deveria }(\mathrm{m}) \text { estar } \\
\text { informado(s) sobre as atividades } \\
\text { desenvolvidas dos seus estudantes. }\end{array}$ & $\begin{array}{l}2 \\
2,3 \%\end{array}$ & $\begin{array}{l}\mathrm{O} \\
\mathrm{O}, \mathrm{O} \%\end{array}$ & $\begin{array}{l}5 \\
5,7 \%\end{array}$ & $\begin{array}{l}41 \\
47,1 \%\end{array}$ & $\begin{array}{l}39 \\
44,8 \%\end{array}$ \\
\hline $\begin{array}{l}\text { 15. O(s) orientador(es) deveria }(\mathrm{m}) \text { orientar trabalhos } \\
\text { de investigação apenas no domínio científico } \\
\text { em que trabalha }(\mathrm{m}) \text {. }\end{array}$ & $\begin{array}{l}1 \\
1,1 \%\end{array}$ & $\begin{array}{l}25 \\
28,7 \%\end{array}$ & $\begin{array}{l}13 \\
14,9 \%\end{array}$ & $\begin{array}{l}25 \\
28,7 \%\end{array}$ & $\begin{array}{l}23 \\
26,4 \%\end{array}$ \\
\hline $\begin{array}{l}\text { 16. } \mathrm{O}(\mathrm{s}) \text { orientador(es) deveria }(\mathrm{m}) \text { ser } \\
\text { promotor(es) de atitudes éticas. }\end{array}$ & $\begin{array}{l}\mathrm{O} \\
\mathrm{O}, \mathrm{O} \%\end{array}$ & $1,1 \%$ & $\begin{array}{l}3 \\
3,4 \% \\
\end{array}$ & $\begin{array}{l}34 \\
39,1 \% \\
\end{array}$ & $\begin{array}{l}49 \\
56,3 \%\end{array}$ \\
\hline $\begin{array}{l}\text { 17. } \mathrm{O}(\mathrm{s}) \text { orientador }(\mathrm{es}) \text { deveria }(\mathrm{m}) \text { gerir e estar a } \\
\text { par dos trabalhos investigativos de todos os seus } \\
\text { estudantes. }\end{array}$ & $\begin{array}{l}0 \\
0,0 \%\end{array}$ & $\begin{array}{l}2 \\
2,3 \%\end{array}$ & $\begin{array}{l}3 \\
3,4 \%\end{array}$ & $\begin{array}{l}37 \\
42,5 \%\end{array}$ & $\begin{array}{l}45 \\
51,7 \%\end{array}$ \\
\hline
\end{tabular}

*1. Discordo totalmente, 2. Discordo, 3. Sem opinião, 4. Concordo, 5. Concordo totalmente

Tabela 3 - Gestão do processo de investigação 
Obtivemos um nível de concordância em todos os itens acima de 89\%, em média, com exceção dos itens 3, 10 e 15 onde existe uma maior dispersão nas respostas. Esta dispersão pode mostrar discordância ou dúvidas sobre o facto: i) dos estudantes solicitarem sempre referências bibliográficas aos seus orientadores, ii) dos estudantes prestarem relatórios regulares aos orientadores, e iii) dos orientadores deverem orientar somente no domínio científico em que trabalham.

\subsection{Em relação ao Interface e à Navegabilidade do IARS ${ }^{\circledR}$}

Tratando-se de uma ferramenta em fase de consolidação, questionámos os sujeitos sobre a interface do $\operatorname{IARS}^{\circledR}$, seu impacto inicial na aprendizagem das suas funcionalidades, dificuldades sentidas e flexibilidade do sistema. Pela Tabela 4 é fácil perceber ofeedback positivo através do alto índice de concordância com as frases de 1 a 8, e de discordância quando se questionou negativamente sobre a organização, a dificuldade de utilização e os sentimentos de frustração ao usar a plataforma online IARS ${ }^{\circledR}$.

\begin{tabular}{|c|c|c|c|c|c|}
\hline & $\mathbf{1}^{*}$ & 2 & 3 & 4 & 5 \\
\hline 1. Foi fácil aprender a usar o IARS ${ }^{\circledR}$. & $\begin{array}{l}0 \\
0,0 \%\end{array}$ & $\begin{array}{l}1 \\
1,1 \%\end{array}$ & $\begin{array}{l}8 \\
9,2 \%\end{array}$ & $\begin{array}{l}49 \\
56,3 \%\end{array}$ & $\begin{array}{l}29 \\
33,3 \%\end{array}$ \\
\hline $\begin{array}{l}\text { 2. A forma como se pode navegar entre as } \\
\text { várias partes do IARS }{ }^{\circledR} \text { é clara. }\end{array}$ & $\begin{array}{l}0 \\
0,0 \%\end{array}$ & $\begin{array}{l}4 \\
4,6 \%\end{array}$ & $\begin{array}{l}9 \\
10,3 \%\end{array}$ & $\begin{array}{l}55 \\
63,2 \%\end{array}$ & $\begin{array}{l}19 \\
\mathbf{2 1}, \mathbf{8} \%\end{array}$ \\
\hline $\begin{array}{l}\text { 3. Os ícones do IARS }{ }^{\circledR} \text { eram de fácil leitura e } \\
\text { compreensão. }\end{array}$ & $\begin{array}{l}1 \\
1,1 \%\end{array}$ & $\begin{array}{l}\mathrm{O} \\
\mathrm{O}, \mathrm{O} \%\end{array}$ & $\begin{array}{l}9 \\
10,3 \%\end{array}$ & $\begin{array}{l}53 \\
60,9 \%\end{array}$ & $\begin{array}{l}24 \\
27,6 \%\end{array}$ \\
\hline $\begin{array}{l}\text { 4. Do ponto de vista estético, as formas } \\
\text { de representação da informação são } \\
\text { visualmente agradáveis. }\end{array}$ & $\begin{array}{l}\mathrm{O} \\
\mathrm{O}, \mathrm{O} \%\end{array}$ & $2,3 \%$ & 7 & $\begin{array}{l}54 \\
62,1 \%\end{array}$ & $\begin{array}{l}24 \\
27,6 \%\end{array}$ \\
\hline $\begin{array}{l}\text { 5. Senti que o IARS }{ }^{\circledR} \text { era metodologicamente } \\
\text { flexível na sua utilização. }\end{array}$ & $\begin{array}{l}\mathrm{O} \\
\mathrm{O}, \mathrm{O} \%\end{array}$ & $\begin{array}{l}2 \\
2,3 \%\end{array}$ & $\begin{array}{l}11 \\
12,6 \%\end{array}$ & $\begin{array}{l}51 \\
58,6 \%\end{array}$ & $\begin{array}{l}23 \\
26,4 \%\end{array}$ \\
\hline $\begin{array}{l}\text { 6. O IARS }{ }^{\circledR} \text { pareceu adequado para elaborar } \\
\text { um projeto e desenvolver a investigação. }\end{array}$ & $\begin{array}{l}\mathrm{O} \\
\mathrm{O}, \mathrm{O} \%\end{array}$ & $\begin{array}{l}0 \\
0,0 \%\end{array}$ & $\begin{array}{l}6 \\
6,9 \%\end{array}$ & $\begin{array}{l}48 \\
55,2 \%\end{array}$ & $\begin{array}{l}33 \\
\mathbf{3 7}, \mathbf{9} \%\end{array}$ \\
\hline $\begin{array}{l}\text { 7. Senti-me estimulado(a) ao usar o } \\
\text { IARS }^{\circledR} \text {. }\end{array}$ & $\begin{array}{l}1 \\
1,1 \%\end{array}$ & $\begin{array}{l}3 \\
3,4 \%\end{array}$ & $\begin{array}{l}6 \\
6,9 \%\end{array}$ & $\begin{array}{l}48 \\
55,2 \%\end{array}$ & $\begin{array}{l}29 \\
33,3 \%\end{array}$ \\
\hline $\begin{array}{l}\text { 8. A minha reação na utilização do } \mathrm{IARS}^{\circledR} \text { foi } \\
\text { boa. }\end{array}$ & $\begin{array}{l}0 \\
0,0 \%\end{array}$ & $\begin{array}{l}3 \\
3,4 \%\end{array}$ & $\begin{array}{l}3 \\
3,4 \%\end{array}$ & $\begin{array}{l}44 \\
50,6 \%\end{array}$ & $\begin{array}{l}\mathbf{3 7} \\
\mathbf{4 2}, \mathbf{5} \%\end{array}$ \\
\hline $\begin{array}{l}\text { 9. A organização da informação no IARS }{ }^{\circledR} \\
\text { era confusa. }\end{array}$ & $\begin{array}{l}30 \\
34,5 \%\end{array}$ & $\begin{array}{l}48 \\
55,2 \%\end{array}$ & $\begin{array}{l}3 \\
3,4 \%\end{array}$ & $\begin{array}{l}6 \\
6,9 \%\end{array}$ & $\begin{array}{l}\mathrm{O} \\
\mathrm{o}, \mathrm{O} \%\end{array}$ \\
\hline 10. Senti que o IARS ${ }^{\circledR}$ é difícil de utilizar. & $\begin{array}{l}34 \\
39,1 \%\end{array}$ & $\begin{array}{l}43 \\
49,4 \%\end{array}$ & $\begin{array}{l}6 \\
6,9 \%\end{array}$ & $\begin{array}{l}4 \\
4,6 \%\end{array}$ & $\begin{array}{l}\mathrm{O} \\
\mathrm{o}, \mathrm{O} \%\end{array}$ \\
\hline 11. Senti-me frustrado(a) ao usar o IARS ${ }^{\circledR}$. & $\begin{array}{l}46 \\
52,9 \%\end{array}$ & $\begin{array}{l}32 \\
36,8 \%\end{array}$ & $\begin{array}{l}3 \\
3,4 \%\end{array}$ & $\begin{array}{l}4 \\
4,6 \%\end{array}$ & $\begin{array}{l}2 \\
2,3 \%\end{array}$ \\
\hline
\end{tabular}

* 1 . Discordo totalmente, 2. Discordo, 3. Sem opinião, 4. Concordo, 5. Concordo totalmente

Tabela 4 - Opinião sobre a Interface do IARS ${ }^{\circledR}$ 
Assim é possível inferir que os utilizadores consideraram o sistema IARS ${ }^{\circledR}$ fácil de utilizar, agradável, flexível, estimulante, claro e de fácil compreensão em termos organizacionais. Aprofundámos também outras dimensões relacionadas à navegabilidade do IARS ${ }^{\circledR}$, como apresentado na Tabela 5 .

\begin{tabular}{|c|c|c|c|c|c|}
\hline & $\mathbf{1}^{*}$ & 2 & 3 & 4 & 5 \\
\hline $\begin{array}{l}\text { Senti dificuldade, do ponto de vista de conhecimento, } \\
\text { na escrita dos conteúdos dos vários campos do } \\
\text { IARS }{ }^{\circledR} \text {. }\end{array}$ & $\begin{array}{l}22 \\
25,3 \%\end{array}$ & $\begin{array}{l}39 \\
44,8 \%\end{array}$ & $\begin{array}{l}15 \\
17,2 \%\end{array}$ & $\begin{array}{l}11 \\
12,6 \%\end{array}$ & $\begin{array}{l}0 \\
0,0 \%\end{array}$ \\
\hline $\begin{array}{l}\text { Na realização das tarefas de preenchimento dos } \\
\text { campos os erros ocorridos eram de fácil } \\
\text { resolução. }\end{array}$ & $\begin{array}{l}0 \\
0,0 \%\end{array}$ & $\begin{array}{l}3 \\
3,4 \%\end{array}$ & $\begin{array}{l}30 \\
34,5 \%\end{array}$ & $\begin{array}{l}41 \\
47,1 \%\end{array}$ & $\begin{array}{l}13 \\
14,9 \%\end{array}$ \\
\hline O sistema IARS ${ }^{\circledR}$ tende a ser confuso. & $\begin{array}{l}\mathbf{2 8} \\
\mathbf{3 2 , 2} \%\end{array}$ & $\begin{array}{l}48 \\
55,2 \%\end{array}$ & $\begin{array}{l}6 \\
6,9 \%\end{array}$ & $\begin{array}{l}4 \\
4,6 \%\end{array}$ & $\begin{array}{l}1 \\
1,1 \%\end{array}$ \\
\hline O sistema IARS ${ }^{\circledR}$ é confiável. & $\begin{array}{l}\mathrm{o} \\
\mathrm{o}, \mathrm{0} \%\end{array}$ & $\begin{array}{l}4 \\
4,6 \%\end{array}$ & $\begin{array}{l}22 \\
25,3 \%\end{array}$ & $\begin{array}{l}44 \\
50,6 \%\end{array}$ & $\begin{array}{l}17 \\
19,5 \%\end{array}$ \\
\hline A velocidade do sistema IARS ${ }^{\circledR}$ é lenta. & $\begin{array}{l}14 \\
16,1 \%\end{array}$ & $\begin{array}{l}\mathbf{5 0} \\
\mathbf{5 7}, 5 \%\end{array}$ & $\begin{array}{l}13 \\
14,9 \%\end{array}$ & $\begin{array}{l}8 \\
9,2 \%\end{array}$ & $\begin{array}{l}2 \\
2,3 \%\end{array}$ \\
\hline Senti necessidade da função de "Ajuda". & $\begin{array}{l}17 \\
19,5 \%\end{array}$ & $\begin{array}{l}32 \\
36,8 \%\end{array}$ & $\begin{array}{l}17 \\
19,5 \%\end{array}$ & $\begin{array}{l}16 \\
18,4 \%\end{array}$ & $\begin{array}{l}5 \\
5,7 \%\end{array}$ \\
\hline $\begin{array}{l}\text { As tarefas de preenchimento dos campos são } \\
\text { realizadas de maneira simples. }\end{array}$ & $\begin{array}{l}\mathrm{o} \\
\mathrm{o}, \mathrm{0} \%\end{array}$ & $\begin{array}{l}2 \\
2,3 \%\end{array}$ & $\begin{array}{l}4 \\
4,6 \%\end{array}$ & $\begin{array}{l}60 \\
69,0 \%\end{array}$ & $\begin{array}{l}21 \\
24,1 \%\end{array}$ \\
\hline $\begin{array}{l}\text { Consigo recordar o nome e as funções de } \\
\text { comandos. }\end{array}$ & $\begin{array}{ll}2 \\
2,3 \%\end{array}$ & $\begin{array}{l}7 \\
8,0 \%\end{array}$ & $\begin{array}{l}18 \\
20,7 \%\end{array}$ & $\begin{array}{l}54 \\
62,1 \%\end{array}$ & $\begin{array}{l}6 \\
6,9 \%\end{array}$ \\
\hline $\begin{array}{l}\text { Explorei as funcionalidades das ferramentas ou } \\
\text { comandos do IARS }{ }^{\circledR} \text { por tentativa e erro. }\end{array}$ & $\begin{array}{l}4 \\
4,6 \%\end{array}$ & $\begin{array}{l}18 \\
20,7 \%\end{array}$ & $\begin{array}{l}16 \\
18,4 \%\end{array}$ & $\begin{array}{l}47 \\
54,0 \%\end{array}$ & $\begin{array}{l}2 \\
2,3 \%\end{array}$ \\
\hline
\end{tabular}

*1. Discordo totalmente, 2. Discordo, 3. Sem opinião, 4. Concordo, 5. Concordo totalmente

Tabela 5 - Opinião sobre a Navegabilidade do IARS ${ }^{\circledR}$

Embora o $\operatorname{IARS}^{\circledR}$ seja uma ferramenta para apoiar a realização de diversas tarefas no processo de desenvolvimento e gestão da investigação, ela não substitui as dificuldades e desafios inerentes ao processo. Por isso, questionámos se a ferramenta não criava dificuldades acrescidas no preenchimento de campos e tarefas. É possível inferir pela Tabela 5 que os participantes não sentiram dificuldade na escrita de conteúdos (70.1\%), consideraram as tarefas de preenchimento simples (93\%), conseguiam recordar as funções do $\operatorname{IARS}^{\circledR}(69 \%)$ e acharam fácil resolver problemas (62\%). Consideraram o sistema confiável (70.1\%) e rápido (73.6\%). Embora 56.3\% tenham reconhecido que não sentiram necessidade da função "Ajuda", é neste item que existe uma maior dispersão de respostas.

Ao desenvolvermos o software, era nosso objetivo criar um sistema que fosse fácil de compreender sem necessidade de manuais e ajudas extras. Esta intencionalidade é confirmada por $56.3 \%$ que exploraram as funcionalidades e comandos do IARS ${ }^{\circledR}$ por tentativa e erro. Com base nestas respostas podemos afirmar que o IARS ${ }^{\circledR}$ possui uma boa e autónoma navegabilidade. 


\section{Conclusões}

Quando refletimos acerca dos desafios que o processo de investigação implica, devemos reconhecer a necessidade de estratégias e ferramentas devidamente coordenadas para o sucesso deste processo. A literatura internacional aponta para vários dilemas e desafios que se colocam. Estes desafios situam-se no âmbito: i) das questões de investigação e da sua fundamentação teórica ii) das dificuldades técnicas e metodológicas, iii) da orientação e interação entre os orientadores e seus orientandos, iv) dos aspetos da inteligência cognitiva e emocional dos envolvidos, v) da gestão geral das etapas do processo investigativo, e vi) das prerrogativas institucionais e legais.

Neste artigo apresentámos uma ferramenta que se constitui também numa estratégia para dar resposta a muitos destes desafios da investigação - a plataforma IARS ${ }^{\circledR}$ (www. ia-rs.com). Sendo um sistema relativamente recente, procedemos a uma avaliação com base num conjunto de utilizadores que participaram nos Workshops sobre o IARS ${ }^{\circledR}$. Mesmo sendo uma avaliação inicial, foi possível obter algumas informações valiosas sobre os primeiros impactos do sistema nestes participantes. As conclusões parciais obtidas podem ser sumariamente especificadas como:

- Os participantes compreenderam as potencialidades do sistema, principalmente para a elaboração e desenvolvimento dos projetos e para a comunicação com o orientador.

- Os participantes, sejam orientadores ou orientados, não expressaram opiniões diferentes em relação aos vários itens investigados.

- Não apontaram dificuldades relevantes na utilização do IARS ${ }^{\circledR}$.

- Os utilizadores acharam o sistema IARS ${ }^{\circledR}$ fácil de utilizar, agradável, flexível, estimulador, claro e de fácil compreensão em termos organizacionais.

- No geral, os participantes se declararam entusiasmados com o sistema IARS ${ }^{\circledR}$.

- Mostraram discordância ou dúvidas sobre o facto: i) dos estudantes solicitarem sempre referências bibliográficas aos seus orientadores, ii) dos estudantes prestarem relatórios regulares aos orientadores, e iii) dos orientadores orientarem fora do domínio científico em que trabalham.

- As duas dificuldades mais apontadas no processo de investigação foram: i) criar e manter a coerência interna de um projeto, e ii) formular questões de investigação.

- Não foram apontadas maiores dificuldades na interface e navegabilidade do IARS $^{\circledR}$.

Neste trabalho apontamos como principal limitação o pouco tempo de utilização do sistema IARS ${ }^{\circledR}$ antes de os participantes responderem a um questionário de avaliação. Portanto estas conclusões são importantes somente para obter uma visão inicial dos orientadores e orientandos que participaram dos Workshops. Compreendemos que temos outros desafios de investigação e análise de usabilidade e User Experience, bem como de outros fatores que o trabalho suscitou (Martins, Alexandra \& Rocha, 2013). Esperamos aprofundar a investigação e as repercussões em contexto de utilizações reais e mais prolongadas do sistema IARS ${ }^{\circledR}$ e suas implicações na qualidade da investigação. 


\section{Agradecimentos}

Este trabalho é financiado por Fundos Nacionais através da FCT - Fundação para a Ciência e a Tecnologia, no âmbito do projeto Inovação Didática no Processo de Orientação e Desenvolvimento de Projetos de Investigação do processo n. ${ }^{\circ}$ 57/ID/2014, e pelo Centro de Investigação Didática e Tecnologia na Formação de Formadores (CIDTFF).

\section{Referências}

Baptista, A. V. (2011). Challenges to Doctoral Research and Supervision Quality: a Theoretical Approach. Procedia Soc. Behav. Sci., 15, 3576-3581.

Agu, N.; C. O. Odimegwu, C. O. (2014) Doctoral dissertation supervision: indentification ande evaluation of models. Educ. Res. Int., (pp. 1-9).

Alarcão, I. (2014). Dilemas do jovem investigador. Dos 'dilemas' aos problemas,” In Costa, P. Neri de Souza, F.; Neri de Souza, D. (Eds.) Investigação Qualitativa: inovação, dilemas e desafios, Vol.1 (pp. 103-123).

Beer, M.; Mason, R. B. (2009) Using a blended approach to facilitate postgraduate supervision. Innov. Educ. Teach. Int., vol. 46, no. 2, pp. 213-226.

Calma, A. (2007). Postgraduate supervision in Philippines: setting the research agenda. Asia-Pacific Educ. Res., vol. 16, no. 1, (pp. 91-100).

Dysthe, O. (2002) Professors as mediators of academic text cultures: An interview study with advisors and master's degree students in three disciplines in a Norwegian University. Writ. Communication, vol. 19, no. 4, pp. 493-544.

Escudeiro, P; Bidarra, J. (2008) Qualitative evaluation framework. RISTI - Revista Ibérica de Sistemas e Tecnologias de Informação, no.1, pp. 16-27

Gurr, G. M. (2001) Negotiating the 'Rackety Bridge'-a dynamic model for aligning supervisory style with research student development. High. Educ. Res. Dev., vol. 20, no. 1, pp. 81-92.

Lessing, A. C.; Schulze, S. (2002) Postgraduate Supervision and Academic Support: Students' Perceptions. South African J. High. Educ., vol. 12, no. 2, pp. 139-149.

Mainhard, T.; Van der Rijst, R.; Wubbels, T.(2009) A model for the supervisor-doctoral student relationship. Higher Education, vol. 58, no. 3, pp. 359-373.

Martins, A. I.; Alexandra, Q. \& Rocha. N. P. (2013) Avaliação de usabilidade: uma revisão sistemática da literatura. RISTI - Revista Ibérica de Sistemas e Tecnologias de Informação, no. 06, pp. 31-44. doi: 10.4304/risti.11.31-43

Neri de Souza, F.; Neri de Souza, D.; Costa, A. (2014) Importância do questionamento no processo de investigação qualitativa, In Costa, A, Neri de Souza, F., Neri de Souza, D. (Eds.); Investigação qualitativa: inovação, dilemas e desafios, $1^{\mathrm{a}}$ ed. Aveiro, Portugal (pp. 125-145). 
Sidhu, G. K.; Kaur, S.; Fook, C. Y. \& Yunus, F. W.(2014) Postgraduate supervision: comparing student perspectives from Malaysia ande the United Kingdom. Procedia Soc. Behav. Sci., pp. 151-159.

Phillips, E. M., \& Derek, S. P. (2005). How to get a PhD: A handbook for students and their supervisors (Fourth.). Open University Press.

Thompson, D. R.; Kirkman, S., Watson, R. \& Stewart, S. (2005) Improving Research Supervision in Nursing. Nurse Educ. Today, vol. 25, pp. 283-290. 